\title{
Palobra, palabra que obra
}

Recepción: 17/09/2012

\author{
Dora Piñeres De la Ossa ${ }^{1}$ \\ Universidad de Cartagena (Colombia) \\ dorapineres21@yahoo.com
}

Javier Hernández García ${ }^{2}$

Universidad de Cartagena (Colombia)

politicartagena@gmail.com

Estella Simancas Mendoza ${ }^{3}$

Universidad de Cartagena (Colombia)

essime1903@hotmail.com

Evaluación: 04/10/2012

Aceptación: 03/11/2012

Artículo de Reflexión

DOI: http://dx.doi.org/ 10.9757/Rhela.20.06

\section{RESUMEN}

Palobra, palabra que obra, es una revista científica en el área de Ciencias Sociales, Humanas y de Educación, dirigida a profesionales, académicos y público en general, publicada anualmente por la facultad de Ciencias Sociales y Educación de la Universidad de Cartagena(Colombia), cuya misión y relevancia es el fortalecimiento de sus procesos investigativos, aportando a la popularización del conocimiento en temas como la construcción socio cultural de lo local, regional, las estructuras de poder en contextos sociales, grupales, estatales, intervención social, trabajo social y contexto, universidad y transformaciones sociales, familia, género, convivencia, desarrollo social y humano, inclusión y exclusión socio política, procesos y actores en la construcción de lo público y lo privado, imaginarios, representaciones y discursos, territorio y poblamiento en la región Caribe, pobreza, ciencia y tecnología, sociedad e innovación, educación, comunicación y cultura, en el con- texto local, regional, nacional e internacional. Indexada en categoría $C$, índice bibliográfico Nacional Publidex-Colciencias y en el Sistema Regional de Información en Línea para Revistas Científicas de América Latina, el Caribe, España y Portugal-Latindex. Actualmente comprometida con los procesos de internacionalización e integración a sistemas de información y a la espera de su revaloración en la Red de Revistas científicas de América Latina, el Caribe, España y Portugal (Redalyc). Hace parte del convenio de colaboración RHELA para la realización de actividades editoriales conjuntas.

Palabras clave: Revista Historia de la Educación Latinoamericana, Palobra, palabra, construcción socio cultural, contextos, intervención social, universidad, transformaciones sociales, desarrollo social, representaciones, inclusión exclusión socio política, familia, género, cultura, comunicación.

1 Profesora de la Universidad de Cartagena, Facultad de Ciencias Sociales y Educación, Doctora en Ciencias Sociales y Educación RUDECOLOMBIA (UPTC), Coordinadora de la Maestría en Educación - SUE Caribe y Editora de laRevista Palobra.

2 Docente Investigador de la Facultad de Ciencias Sociales y Educación de la Universidad de Cartagena, especialista en Métodos y Técnicas Avanzadas de Investigación Social. Hace parte del comité editorial y miembro fundador de la Revista Palobra.

3 Magíster en Historia Universidad Pedagógica y Tecnológica de Colombia. Profesora Universidad de Cartagena. 


\author{
Wordk, word that works ${ }^{1}$
}

ABSTRACT

Palobra, palabra que obra, is a scientific journal in the area of social sciences, humanism and education, addressed to professionals, academics and public in general. It is published by the Faculty of social sciences and education from the University of Cartagena, Colombia, whose mission and relevance is strengthening related to the research process, it contributes to the popularization of knowledge on issues such as the construction of the local and regional socio-cultural, the structures of power in contexts such as: social groups, state, social intervention, social work, university and social transformations, family, gender, coexistence, social and human development, inclusion and exclusion-partner policy, processes and actors in the construction of public and private, imaginary, representations and discourses, territory and population in the Caribbean region, poverty, science and technology, society and innovation, education, communication and culture, in the local, regional, national and international contexts. Index-linked in C category, bibliographical National index Publidex - Colciencias and in the Regional System of Information in Line for Scientific Magazines of Latin America, the Caribbean, Spain and Portugal-Latindex. Currently, it is engaged in the processes of internationalization and integration to systems information and in expectation of its revaluation in the Network of scientific Magazines of Latin America, the Caribbean, Spain and Portugal (Redalyc). It does part of the agreement of collaboration RHELLA for the achievement of publishing joint activities.

keywords: History of Latin American Education Journal, Palobra, word, socio-cultural construction, contexts, social intervention, university, social transformations, social development, representations, inclusion and exclusion in policies, family, gender, culture, communication.
Palobra, palabra que obra

\section{RESUMO}

Palobra, palabra que obra, é uma revista científica na área de Ciências Sociais, Humanas e de Educação, dirigida a profissionais, acadêmicos e público em geral, publicada anualmente pela faculdade de Ciências Sociais e Educação da Universidade de Cartagena, Colômbia, cuja missão e relevância é o fortalecimento de seus processos de pesquisa, contribuindo com a popularização do conhecimento em temas como a construção sócio cultural do local, regional, as estruturas de poder em contextos sociais, grupais, estatais, intervenção social, Trabalho Social e contexto, universidade e transformações sociais, família, gênero convivência, desenvolvimento social e humano, inclusão e exclusão sociopolítica, processos e atores na construção do público e do privado, imaginários, representações e discursos, território e povoamento na região do Caribe, pobreza, ciência e tecnologia, sociedade e inovação, Educação, comunicação e cultura, no contexto local, regional, nacional e internacional. Indexada na categoria $C$, índice bibliográfico Nacional Publidex-Colciencias e no Sistema Regional de Informação em Linha para Revistas Científicas da América Latina, Caribe, Espanha e Portugal-Latindex. Atualmente comprometida com os processos de internacionalização e integração a sistemas de informação e a espera de sua reavaliação na Red de Revistas científicas de América Latina, Caribe, Espanha e Portugal (Redalyc). Faz parte do convenio de colaboração RHELA para a realização de atividades editoriais conjuntas.

Palavras-chave:Revista História da Educação Latino-americana, Palobra, palavra, construção sócio cultural, contextos, intervenção social, universidade, transformações sociais, desenvolvimento social, representações, inclusão exclusão sócio política, familia, gênero, cultura, comunicação.

1 It is the name of a magazine; the translation does not match into English. 


\section{INTRODUCCIÓN}

El presente Artículo de revisión, responde a la convocatoria de la Revista Historia de la Educación Latinoamericana, dedicada a la trayectoria de las revistas en el campo de la Historia y la Educación, con motivo de la celebración de los 15 años de RHELA. El artículo da cuenta de la construcción analítica, interpretativa y crítica de las tendencias y líneas temáticas de investigación que se asumen en la revista Palobra, palabra que obra en sus diversas ediciones desde el año 2000 al 2013; las fuentes primarias desde donde se sustenta su escritura son los registros de las ediciones originales y cada uno de los artículos que sustentan las publicaciones, de tal modo que las referencias de textos son fundamentales para validar las reflexiones que se hacen en torno a las tendencias y resultados de investigación a las cuales le apuesta la revista.

\section{Antecedentes e historia de la revista Palobra, palabra que obra.}

La Universidad de Cartagena y su joven Facultad de Ciencias Sociales y Educación diseñaron, en el año 2000, una estrategia activa de vinculación con los intereses y desenvolvimientos de las dinámicas sociales con la construcción de saberes cada vez más rigurosos y pertinentes y con los requerimientos del cambio y del progreso de la región Caribe. Desde estos intereses se concibió la estructura particular de la revista Palobra, palabra que obra, con ejes temáticos definidos, por una parte, hacia asumir asuntos del orden social, político y cultural más relevantes del entorno local y regional y, por otra, de manera integrada, que planteara discusiones, problemáticas y realidades relacionadas con las ciencias de la educación, exigencias, retos y perspectivas. Bajo esa directriz, el primer número de publicación de la revista Palobra -septiembre del año 2000- se ubica dentro del movimiento de desarrollo y renovación que por entonces se cumplía en la antigua Facultad de Trabajo Social, la que, a partir de 1994, se transforma en una nueva Facultad. Movimiento que se manifestaba en los diversos ámbitos y derroteros que su misión institucional establecía, entre otros, aquellos que tenían que ver con el planeamiento, la formulación y puesta en servicio de nuevos programas de pregrado y posgrado y de educación continuada, en la intensificación y ampliación de cobertura de las redes de servicios de extensión en el medio local y regional, y, particularmente, en la formalización y puesta en acción de los grupos de investigación Colciencias como expresión de una, cada vez más rigurosa, configuración en la Facultad de comunidades científico-inves- 
tigativas que cualificaban progresivamente tanto el proceso de construcción colectiva del conocimiento como las labores concomitantes de docencia y formación.

En el contexto global de transformaciones, que desde entonces caracterizaba a la región, la Facultad de Ciencias Sociales y Educación había asumido con la mayor seriedad y diligencia el compromiso de apoyar, desde la investigación, la docencia y el servicio educativo, la formación de una masa crítica de saberes y de una comunidad científica profesional que vinculara las nuevas generaciones a la tarea de construcción de un nuevo modelo de sociedad. Así, entre otras cosas, se consideró que esa misión fundamental se cumplirá poniendo todo el empeño en convertir el espacio académico de la Facultad en el lugar de encuentro, la contrastación y la validación de las diferencias, y la búsqueda de los acuerdos en el campo de las ideas, del saber y de la invención, valorando y haciendo valorar tano el descubrimiento que renueva como la tradición que sustenta y radica. En ese escenario fue que se consideró oportuno poner a disposición de la comunidad esta propuesta de comunicación y encuentro, como se afirmó en el primer artículo Editorial, "La publicación de esta Revista, forma parte de nuestra estrategia de activa circulación con los intereses y desenvolvimientos de las dinámicas sociales, con la construcción de saberes cada vez más rigurosos y pertinentes, y con los requerimientos del cambio y el progreso de nuestra región". ${ }^{4}$

\section{Los Inicios}

En este escenario en transformación, donde la vieja expectativa de contar con un medio de divulgación y debate públicos desde la Facultad se estaba produciendo, a través del Departamento de Investigación y del grupo de investigación Cultura, Ciudadanía y Poder en Contextos Locales, liderado por la socióloga profesora, Carmen Cabrales Vargas, la tarea de promover y gestionar lo pertinente, en lo administrativo, lo financiero y, sobre todo, en lo científico-académico para la realización de este sueño. La respuesta fue positiva desde todos los ámbitos de la Universidad y de la Facultad. En particular, entre el cuerpo de docentes investigadores de la Facultad que convirtieron progresivamente en un reto personal y profesional responder a la convocatoria que se hacía desde el naciente Comité Editorial de lo que fue nominada Revista Palobra.

4 Carmen Cabrales. Editorial. Revista Palobra, No.1, (2000): 5-7. 
Así fue que durante el periodo rectoral 2000-2003 se logró sacar a la luz el primer número orientado por el Comité Editorial, conformado por los docentes de la Facultad Carmen Cabrales Vargas, Kenia Victoria Cogollo y Javier Hernández García. Los articulistas de ese primer número, con la sola excepción de Yolanda Puyana, investigadora de la Universidad Nacional, provenían de la Facultad, junto con colaboradoras de otras Facultades de la Universidad. Se carecía de un Comité científico, sin pares evaluadores externos para la valoración formal y científica de las colaboraciones, de tal manera que la valoración académica de los artículos, así como la edición, corrección de estilo, consecución del material gráfico e, incluso, el control de calidad litográfico, correspondió al mismo equipo editorial. Desde ese número inicial se perfiló la estructura básica de la Revista, de periodicidad anual, que ha servido de matriz para los ulteriores y constantes perfeccionamientos tanto de forma como de contenidos. Una página Editorial, donde se incluía el pensamiento del Consejo editorial sobre el número publicado y se presentaban los artículos. Una Página de Opinión, que se convirtió en un corto espacio de reflexión de fondo sobre algún o algunos de los temas tratados en ese número de la Revista, aportado por algún docente investigador de la Facultad o un tercero invitado por el Comité Editorial. Luego, el grueso del material lo constituía el conjunto de artículos científicos que abordaban temáticas en las que los colaboradores estaban trabajando en su ejercicio docente-investigativo universitario regular. No se distinguía entonces el formato epistemológico, metodológico o argumentativo del artículo, publicándose indistintamente, y con un peso relativo aleatorio dentro del total de artículos publicados en los primeros números, entre artículos productos de investigación, artículos que adelantaban una reflexión, ensayos o crónicas académicas.

En los números iniciales de Palobra (del 1 al 5) se presentaron resultados de investigaciones terminadas o en proyectos en ejecución, o en ponencias presentadas a congresos científicos nacionales, que desde escenarios locales, regionales y nacionales estuvieron centrados en estudios de género ${ }^{5}$, familia ${ }^{6}$,

5 Gloria Bonilla Vélez, "Bellas, Casadas, madres y solteras, imágenes femeninas en el Caribe colombiano a comienzos del siglo XX", Revista Palobra, No. 1 (2000): 66-78; Yolanda Puyana, “Cómo se convierten en mujeres las niñas del norte de Bolívar", Revista Palobra, palobra que obra. No. 1 (2000):25-39; Dora Piñeres de la Ossa,"El ingreso de la mujer a la universidad, entre discursos y debates: el caso de Paulina Beregoff. La primera universitaria en Colombia”, Revista Palobra, No. 3 (2002): 19-32.

6 María del Pilar Morad de Martínez,"La construcción sociocultural de la maternidad y la paternidad en Cartagena de indias", Revista Palobra, palabra que obra.No. 1 (2000): 16-24; María del Pilar Morad de Martínez,"Familias: espacios con posibilidades de construir y deconstruir ideas de equidad entre los géneros”, Revista Palobra No. 3 (2002): 123-131. 
universidad y reformas, problemáticas relacionadas con las ciencias de la educación ${ }^{7}$, ciudadanía y cultura ${ }^{8}$ y pedagogía del conflicto. ${ }^{9}$

\section{Perfilamiento de una revista científica: de la revista de artículos sueltos a una revista temática indexada}

El proceso de indización de la Revista Palobra ante Colciencias-Publindex se inicia desde el año 2005, y cumplió varias etapas. Un primer paso fue el de la formulación y cumplimiento de un conjunto riguroso de requisitos y requerimientos de forma y de contenido que debían cumplir todos los materiales publicados por esta, ajustándose a las normativas vigentes en Colombia, aplicadas a revistas académico-científicas. Requisitos y requerimientos constituidos en condiciones necesarias para su inclusión en la lista de revistas científicas indexadas por este ente nacional. Ese proceso lo inicia el Comité Editorial, con relacióna la normativa nacional y al tiempo, ajustándola a las condiciones particulares del contexto académico y cultural de donde emergía Palobra, con un documento que se publica por primera vez en el número correspondiente al año 2006. Allí bajo el título de "Requisitos y Requerimientos de las Colaboraciones para la Revista Palobra" se incluyen lineamientos condicionantes tanto de forma como de contenido que debían cumplir los articulistas interesados en participar de las convocatorias anuales hechas por Palobra.

En primer lugar, se establecían los tipos de artículos que podían ser considerados para su evaluación y eventual publicación en la Revista. Las colaboraciones presentadas al Comité Editorial de Palobra deberían tener un formato que se ajustara a la tipología establecida por Publindex-Colciencias. Se privilegiaba, tal como lo exigía Colciencias, los artículos basados en procesos investigativos vigentes o resultados de investigaciones terminadas por el mismo autor del artículo. Eran estos, entonces, los Artículos de Investigación Científica, documento que presentaba resultados originales de proyectos de

7 Dora Piñeres de la Ossa, "El movimiento renovador estudiantil y las reformas universitarias en Colombia, 1920-1930", Revista Palobra, palabra que obra. No. 1 (2000): 78-87; Dora Piñeres, "El ingreso de la mujer", Revista Número Año19-32; Dora Piñeres de la Ossa, "La pedagogía activa en los claustros de la Universidad de Cartagena, la presencia del pedagogo alemán Carl Glockner”, Revista Palobra, palabra que obra. No. 5 (2004): 19-27; Nancy Bolaño, Carmen Cabrales, y Javier Hernández, "Algunos elementos de la construcción de "lo universitario" por los estudiantes de la Universidad de Cartagena", Revista Palobra, palabra que obra. No. 1 (2000): 87-97.

8 Edgar Gutiérrez, "Elementos para una filosofía del universo simbólico y ciudadanía en el contexto de Colombia y Latinoamérica", Revista Palobra, palabra que obra. No. 2 (2001): 12-27; Marco Raúl Mejía, "Hacia una pedagogía del conflicto", Revista Palobra, palabra que obra. No 2 (2001): 60-82.

9 Marco Raúl Mejía,“Hacia una pedagogía del conflicto”, Revista Palobra, palabra que obra. No. 2 (2001): 60-82. 
investigación terminados; y los Artículos de Reflexión, documentos que presentarían resultados de investigación terminada por el autor desde una perspectiva analítica, interpretativa o crítica, sobre un tema específico, recurriendo a fuentes originales. Dentro del tipo de artículos científicos preferentes se encontraban también los Artículos de Revisión, documentos resultados de una investigación terminada por el autor donde se analizan sistematizan e integran los resultados de investigaciones publicadas o no publicadas, sobre un campo temático, con el fin de dar cuenta de avances y tendencias de desarrollo. Se caracteriza por fundamentarse en una revisión bibliográfica rigurosa.

Junto con los anteriores, pero ya no necesariamente vinculados de manera directa a investigaciones en marcha o terminadas por el articulista, se recomendaba a los colaboradores potenciales presentar las siguientes opciones: artículos de Revisión de Tema, que son aquellos en los que sedeberá exponer el resultado de una revisión crítica de la bibliografía sobre un tema que no esté soportada por una investigación científica por parte del autor; así como los documentos de reflexión no derivados inmediatamente de investigación (que comúnmente se clasifican como ensayo); documentos de Trabajo, es decir, aquellos textos que adelantan avances teóricos, metodológicos y empíricos sobre casos específicos provenientes de investigaciones en marcha que se someten a escrutinio público. También se convocaba a los autores de testimonios, entendiéndose por tales tanto las transcripciones de documentos históricos (públicos y privados) no publicados y/o materiales etnográficos (biográficos, relatos, etc.) que se publican (con la debida autorización de las fuentes) para su divulgación; como las crónicas, refiriéndose este tipo de artículos a los documentos que recogieran impresiones e interpretaciones del autor sobre circunstancias contextuales presentes. Finalmente, se mantenían las Notas (Notas de Aquí, Notas de Allá): Notas breves y reseñas informativas sobre eventos de interés científico-académico provenientes de las actividades ordinarias de docencia, investigación y extensión generados por y/o en los que participé la Facultad de Ciencias Sociales y Educación y la Universidad de Cartagena, por intermedio de sus estudiantes, cuerpo docente y administrativo.

Si bien se venía haciendo progresivamente de esa manera, en este número, y por medio de la declaración de principios contenida en el documento que se comenta, Palobra definía como regla universal que la aprobación o no para publicación de los artículos enviados a la Revista recaería totalmente sobre 
los hombros de los conceptos emitidos por los evaluadores científicos externos, que ya para ese número incluía tanto a docentes investigadores externos a la Facultad, algunos de la Universidad de Cartagena, como a evaluadores de Universidades nacionales o regionales, quienes deberían tener en cuenta los criterios de novedad y relevancia social del tema; estructura lógica y coherencia argumentativa y metodológica; coherencia entre los campos temáticos establecidos por Palobra y el propio del artículo; la calidad de la argumentación, orden y lógica de exposición, y la redacción y claridad escritural.

Otro aspecto establecido tenía que ver con los campos temáticos sobre los cuales versarían los artículos, centrados en la pertinencia y relevancia de problemáticas y temáticas del orden regional y de los niveles nacional e internacional, relacionadas con la Construcción sociocultural de lo local y lo regional; estructuras de poder en contextos sociales, grupales y estatales; Intervención, Trabajo social y contexto; ${ }^{10}$ Universidad y transformaciones sociales; Familia, género y cambio social; ${ }^{11}$ convivencia y desarrollo humano; Procesos de inclusión y exclusión socio-política; ${ }^{12}$ procesos y actores en la construcción de lo público y lo privado. Imaginarios, representaciones y discursos; Territorio y poblamiento en la región Caribe; pobreza, desarrollo y territorio;: ${ }^{13}$ Ciencia, tecnología, sociedad e innovación; educación para el sistema nacional de ciencia y tecnología; ${ }^{14}$ Comunicación y cultura; ${ }^{15}$ Comunicación y desarrollo; Comunicación y Educación, temáticas estas objeto de estudio de los grupos de investigación de los docentes de la facultad de Ciencias Sociales y Educación.

10 Kenia Cogollo, Lewis Leon y Berena Vergara Serpa, "Cartagena de Indias y la asociatividad como estrategia competitiva de sobrevivencia en el desarrollo local”, Revista Palobra No. 6 (2005): 28-41; Julio Amezquita López y Erik Baldovino Villacop, "Prospectiva industrial de las mini cadenas productivas hortofrutícolas de Bolívar", Revista Palobra, palabra que obra No.7(2007): 107-118.

11 Hortensia Naizara Rodríguez González, "La violencia intrafamiliar en Cartagena: un asunto de mentalidades", Revista Palobra, palabra que obra. No. 6 (2005): 71-84; Mabel Valencia y Carlos Ospina, "El abordaje de la problemática de la explotación sexual infantil en Cartagena", Revista Palobra, palabra que obra.No. 6 (2005): 137-153.

12 Claudia Mosquera Rosero-Labbé, "Sufrir el desplazamiento forzado para conocer los Derechos: impactos del desplazamiento forzado en mujeres afrocolombianas residentes en Bogotá", Revista Palobra, palabra que obra. No. 6 (2005): 42-53; Edgar Rey Sinning, "Las nuevas liturgias patrióticas: Celebraciones en Santa marta, con ocasión de la elección y posesión de tres presidentes neogranadinos", Revista Palobra, palabra que obra. No. 7 (2006): 76-91.

13 Morelia Pabon Patiño, "Instauración de la Formación ambiental en la Universidad Colombiana", Revista Palobra, palabra que obra. No. 7 (2006): 51-75.

14 Carlos Ospina Bozzi, "Festival ver ciencia Caribe: Un modelo de apropiación social del conocimiento científico regional", Revista Palobra, palabra que obra. No. 7 (2006): 38-50.

15 Javier Hernández García,“Una teoría retórica de la Sociedad”, Revista Palobra, palabra que obra. No. 6 (2005): 104-122. 


\section{De la revista de colegas de la Facultad y la Universidad a la revista de autores de cobertura nacional e internacional}

El primer Comité Editorial fundador se amplía desde el segundo número, y ya para 2002 incluía a colaboradores estrechos desde su inicio, como el investigador sociólogo Edgar Rey Sinning, y al respaldo científico y moral del proyecto, el maestro Orlando Fals Borda, que siempre estuvo atento y apoyando esta aventura intelectual emprendida desde una universidad de su querida provincia Caribe. El grueso de articulistas seguía siendo por entonces el grupo de docentes de la Facultad de Ciencias Sociales y Educación, pero aparecían crecientemente nombres de autores provenientes de las universidades de la región y nacionales. Fue en el cuarto número de Palobra donde empezaron a aparecer autores internacionales introduciendo problemáticas y temáticas sociales de diverso orden con claras sincronías e isotopías con las correspondientemente tratadas por los autores locales y nacionales, dándole a la Revista una cobertura y profundidad de campo creciente, tendencia que se mantiene desde entonces hasta el presente, logrando siempre un equilibrio entre las discusiones microlocalizadas en artículos sobre el entorno cartagenero y regional Caribe, el nacional e internacional, particularmente, sin serlo de manera excluyente, aplicado al ámbito iberoamericano y latinoamericano.

A partir de la edición número 7 correspondiente al año 2006, la Revista se ajusta tanto en su formato como en la organización de su contenido, a los lineamientos establecidos por Colciencias-Publindex. ${ }^{16}$ Por una parte, junto al Comité Editorial, que se ha mantenido desde el año 2001, se incluye un nuevo Comité Científico, conformado por intelectuales y académicos de reconocida trayectoria internacional (de Argentina, Norberto Alayón y Diana Maffia; de Trinidad y Tobago, Lancelot Cowie, de Chile, Leonardo Oneto; de Colombia, Orlando Fals Borda y Javier Ocampo). Un Editor se ocupa de coordinar las tareas del Comité Editorial y los pares evaluadores siguen contando principalmente con docentes investigadores de la Universidad de Cartagena, y la región Caribe y un par de evaluadores nacionales (Universidad de Antioquia y Universidad Nacional, Bogotá).

16 Javier Hernández García,"Difundir, visibilizar, competir”, Revista Palobra, palabra que obra. No.7 (2006): 9-14. 
Sometidos los números publicados en 2006 y 2007 a consideración del comité evaluador de Publindex-Colciencias, consideró este comité que si bien cumplía con una parte de los requerimientos para su indexación, en particular a lo que hace a la proporción de artículos contenidos allí, según la tipología arriba establecida y en las proporciones establecidas por Publindex, había otros requerimientos de carácter formal que aún no se satisfacían (doctores en su comité editorial, por ejemplo). Sin embargo, se cumplían los principales y fundamentales requisitos para su indexación: calidad científica de sus contenidos; calidad editorial de la Revista; estabilidad (7 años continuos de aparición) y visibilidad (a través de una política consistente de divulgación y promoción nacional e internacional de la Revista, primero mano a mano y luego por vía de correo nacional e internacional). Acicateados por la positiva valoración de la Revista ante Colciencias, el Comité editorial que ya había incluido en su Comité desde el 2008, a una antigua colaboradora de la Revista, tanto como articulista como apoyo desde sus inicios del Comité editorial, Liliana Pérez; y en su Comité Científico por Colombia, a María Imelda Ramírez y a Dora Piñeres de la Ossa. Al tiempo que se daba noticia del fallecimiento de su miembro fundador, Orlando Fals Borda.

En este contexto de indexación a partir de la edición número 9 la revista Palobra consolida tendencias temáticas que le apuestan no solo a un diálogo interdisciplinar y transdiciplinario sino que además comparte diversos enfoques, problemas y paradigmas investigativos desde redes de investigación nacionales e internacionales a la redefinición de los escenarios y contextos locales y regionales, fundamentados en estudios de géneros desde la disciplina de la historia y el trabajo social, las representaciones discursivas en intervención social del trabajo social, ${ }^{17}$ configuración de las identidades locales, pobreza y desigualdad social en el contexto urbano, ${ }^{18}$ ciudadanía y

17 Leonardo Oneto Piazze, "De las representaciones discursivas al orden del discurso: Una perspectiva discursiva de la intervención del Trabajo Social”, Revista Palobra, palabra que obra. No. 9 (2008): 27-41; Liliana Pérez Mendoza, "Las Políticas e intervenciones sociales, algunas consideraciones ética", Revista Palobra, palabra que obra No. 11 (2010): 24-38; Juan Masullo Jiménez, "La Genética del discurso del desarrollo", Revista Palobra, palabra que obra. No. 9 (2008): 84-103; Norberto Alayon y María Lorena Molina, "La desigualdad social: Desarrollos y Desafíos del Trabajo Social desde la re conceptualización en América Latina", Revista Palobra, palabra que obra. No. 9 (2008): 35-50.

18 Rina de León Herrera, "Los estudios de pobreza urbana”, Revista Palobra, palabra que obra. No. 9 (2008): 78-98; Camilo Rey Sabogal, "Aproximaciones teóricas y empíricas a la relación de causalidad entre desigualdad y crecimiento: Un análisis para Colombia. 1985-2006”. Año 164-184. 
política, resistencia en territorios fronteras ${ }^{19}$ formas de trabajo y estilos de vida en la primera mitad del siglo XX e imaginarios de familias. ${ }^{20}$

\section{CONCLUSIÓN}

El proceso de perfeccionamiento formal y de contenido de la Revista se mantiene, y se procede de nuevo a solicitar indexación de la Revista ante Publindex-Colciencias. Se presentan los números correspondientes a los años 2007 y 2008 y, por fin, en el año 2009, la editora puede dar la noticia en el número correspondiente a ese año de que la revista ha sido indexada en Publindex, en la categoría C. Se convierte así, en la primera revista académico científica que, exclusivamente respaldada por la Universidad de Cartagena, es integrada a la lista de revistas científicas indexadas de Colombia y la primera en la Costa Caribe colombiana en al área de las ciencias sociales.

Pero al mismo tiempo que se procedía a lograr la indexación nacional, Palobra buscaba reconocimiento internacional. Se escribe en este editorial Palobra, Diez años de calidad

Hoy con orgullo decimos a todos los que de una u otra forma han apoyado esta idea, y a aquellos para quienes cada ejemplar de la Revista se convierte en una invitación por una apuesta hacia una sociedad más incluyente y menos discriminatorio, más justa y solidaria, que nuestra Revista PALOBRA después de cumplir con parámetros de calidad internacional ha sido indizada en las prestigiosas bases de datos CLASE, DIALNET, EBSCOhost, pero además al sistema nacional de Indexación y su Homologación de revistas especializadas de CT + I Publindex de Colciencias y al Sistema Regional de Información en línea para revistas científicas de América Latina, el Caribe, España y Portugal, REDALYC.

19 Alejandro Pimienta Betancourt, "La configuración de la identidad local en la diversidad cultural: El Caso de Caucasia", Revista Palobra, palabra que obra. No. 9 (2008): 60-77; Orlando Durango Rueda, "Estableciendo rastros para la construcción de una persona activa de Derechos. El cuerpo de la violencia en la historia de los Montes de María, Revista Palobra, palabra que obra. No. 9(2008): 121-136; Javier Hernández,"Formas y modos del trabajo en la hacienda tradicional Monte mariana. (1930-1960)", Revista Palobra, palabra que obra. No. 9, (2008): 102-125; Rafael Acevedo Puello, "Apuntes acerca de la ciudadanía política en el Caribe Colombiano: El pensamiento educativo de principios del siglo XX”, Revista Palobra, palabra que obra. No. 9, (2008): 81-101; Sergio Paolo Solano de las Aguas, "Imprentas, Tipógrafos y estilos de vida en el Caribe Colombiano. 1850-1930", Revista Palobra, palabra que obra. No.9, (2008): 126-145.

20 Gloria Bonilla, "La lucha de las mujeres en América latina: Ciudadanía y Derechos", Revista Palobra, palabra que obra. No. 9, (2008): 45-59; Carmenza Jiménez Torrado y Leydi Perneth Pareja "Exigencias metodológicas del abordaje de la realidad social desde una perspectiva de género." Una experiencia en construcción, Revista Palobra, palabra que obra. No. 9 (2008): 99-120; Gloria Bonilla Vélez, Pilar Morad de Martínez, y Anatali Oquendo Lorduy, "Feminidades y maternidades en Cartagena de indias: Entre las innovaciones y los conflictos", Revista Palobra, palabra que obra. No. 10, (2009): 57-72. 
Esto la constituye en la primera revista de la Universidad de Cartagena en llegar a esta meta, constituyéndose en un modelo a seguir de ahora en adelante tanto para la revista misma, como también para las otras revistas de nuestra Alma Mater, que creemos son reflejo de la calidad de nuestra institución.

Apartir de la publicación de la edición 12 y bajo la dirección editorial de Dora Piñeres de la Ossa, la Revista dio cuenta de las exigencias para continuar con los procesos de internacionalización e integración a sistemas de información y a la espera de la revaloración de la red de revistas científicas de América Latina, el Caribe, España y Portugal Redalyc, mostrando y demostrando el interés pleno por mantener la calidad editorial y seguir atendiendo los indicadores internacionales, se dio cumplimiento de la valoración de condiciones de los miembros de comité editorial, las titulaciones de los pares externos.

La participación de pares internacionales se hizo notoria y en las ediciones al cierre de este balance y revisión -12 y 13- se concretan experiencias editoriales de la mano de investigadores como Ricardo Pérez Montfort ${ }^{21}$ y Gabriela Pulido pertenecientes al centro de investigaciones y Estudios Superiores en Antropología CIESAS y el Instituto Nacional de Antropología e Historia, México; Lorenzo Agar Corbinos, ${ }^{22}$ de la Universidad de Chile; David López Jiménez de la Universidad de Huelva, España ${ }^{23}$, consolidando la cultura de las redes y grupos pares con investigadores de la Universidad de Cartagena.

En cuanto a la caracterización de las líneas de análisis representadas en los artículos de la edición 13, se podrían relacionar en cuatro áreas fundamentales de atención: los primeros sobre estudios de género como construcción sociocultural, ${ }^{24}$

21 Ricardo Pérez Montfort y Gabriela Pulido Llano, "Cultura Cubana y Medios de Comunicación en México, 1920-1950", Revista Palobra, palabra que obra. No. 12 (2011): 16-35.

22 Lorenzo Agar Corbinos, "Migraciones posmodernas en Chile: Reflexiones sobre cohesión social y pluralidad cultural, Revista Palobra, palabra que obra. No. 12 (2011): 108-129.

23 David López Jiménez, "La gestión de la imagen subjetiva y objetiva de la empresa en los nuevos canales comerciales", Revista Palobra, palabra que obra. No. 12 (2011): 56-71.

24 Marcela Aguirre Zabala, y Marcela Tejerina,"Entre quejas confiadas y súplicas de amparo: una aproximación a la condición de la mujer en el Rio de la Plata a fines del siglo XVIII"Revista Palobra, palabra que obra. No.13 (2013):18-29; José Wilson Márquez, "Delitos sexuales y práctica judicial en Colombia: 1870-1900. Los casos de Bolívar, Antioquia y Santander", Revista Palobra, palabra que obra. No. 13 (2013):30-49. Antonio Irigoyen,"La familia en la obra de la novelista argentina Juana Manuela Gorriti”, Revista Palobra No. 13 (2013):5 0-69. Guitte Hartog y Ana Lorena Carrillo Padilla, (Universidad Autónoma de Puebla-México: Instituto de Ciencias Sociales y humanidades "Expresiones de la actividad política desde el cuerpo de las mujeres activistas", Revista Palobra, palabra que obra. No.13 (2013): 70-83; María Margarita Echeverri Buritica, Universidad Javeriana, Claudia Pedone, Institut de l'Infància i MónUrbà de Barcelona: "Entre la estigmatización y la restricción". Políticas migratorias y discursos políticos sobre familia, migración, género y generación en países de inmigración y emigración: España y Colombia" Revista Palobra, palabra que obra. No.13 (2013): 84-107; Alex Pérez Álvarez, "Poner el grito en el cielo: Diversidad sexual e identidades de género en familias con prácticas patriarcales en Cartagena de Indias, 2010-2012”, Universidad de 
política, ${ }^{25}$ salud ${ }^{26}$ y turismo. ${ }^{27}$ Un conjunto de temas y asuntos relacionados con estudios sobre género, mujer, prácticas, migraciones; temas decisivos, entre otros, en la afirmación de que se está en un momento histórico de transición de tendencias investigativas que privilegian o evidencian estudios desde la perspectiva de género en los que se revelan los planos de la vida de las personas, la familia, los grupos sociales, las instituciones y de la sociedad en general. Se evidencian nuevos discursos sobre estudios sobre mujer y género, que en nada se parecen a la censura y a la estigmatización de su participación en la esfera de lo público tan afirmante aun, al final del siglo pasado. Estudios que dejan ver categorías de análisis plurales asumidas por los grupos de investigación locales, nacionales e internacionales, asuntos que convergen y solidifican los discursos de las redes y las ciencias sociales en las que está adscrita la Revista Palobra.

Así mismo, Palobra hizo parte del convenio marco entre la Revista Historia de la Educación Latinoamericana, RHELA y otras Revistas, mediante el que se determina el acuerdo de colaboración para la realización de actividades editoriales conjuntas para la difusión y promoción de estas. ${ }^{28}$

Acogiéndose a las directrices de Colciencias, Palobra actualmente está participando en la I Actualización del Índice Bibliográfico Nacional-Publindex 2012, para los procesos de re-indexación y habiendo superado la revisión inicial se encuentra a la espera de los resultados de categorización emitidos por Publindex<http:/ / 201.234.78.173:8084/publindex/>. Como condición para ello, el equipo editor y técnico de la Revista participó el pasado noviembre en una capacitación sobre el manejo de Open Journal Sistem, organizada por Colciencias. Este software permite la agilización del proceso editorial y la participación en las diferentes convocatorias de indexación bibliográfica.

Cartagena "Revista Palobra, palabra que obra. No.13 (2013):108-123; Amparo Montalvo Prieto y Edna Gómez, "Enfermería en Cartagena a inicios del siglo XX (1900-1920)" Universidad de Cartagena. Revista Palobra, palabra que obra. No. 13 (2013): 124-143. Dora Piñeres de la Ossa, "Mujer y Poder: representaciones sociales. Caso de "Beatriz Bechara de Borge, primera rectora Universidad de Cartagena 1988-1990. Revista Palobra, palabra que obra. No. 13(2013): 144-163.

25 Mercedes Posada Meola,"Sistema clientelar, mediaciones y elecciones de alcalde durante el siglo XXI en Cartagena de indias, Revista Palobra, palabra que obra. No. 13 (2013): 164-189.

26 Ana Cristina Quesada y Lidia Isabel Picado,"Aplicación del enfoque de promoción de la salud en la Región Central de Occidente de Costa Rica”. Revista Palobra No. 13(2013):212- 235.

27 Carrillo, Harold, et al.,"El desarrollo de la actividad turística en Cartagena de indias: implicaciones sociales y urbanas. 19431978, integrantes grupo instituto estudios del Caribe, universidad de Cartagena", Revista Palobra, palabra que obra No.13 (2013): 190-211.

28 Revista Historia de la Educación Latinoamericana. Convenio marco entre revista historia de la Educación latinoamericana y otras revistas mediante el que se determina el acuerdo de colaboración para la realización de actividades editoriales conjuntas para la difusión y promoción de estas. Manizales, 31 de agosto 2011. 
Con ello se podrá avanzar en la línea de internacionalización y apertura editorial que se ha propuesto.

El reto del actual comité editorial es alcanzar una categoría superior en la indexación de Publindex, demostrando así que los sueños de los profesores de la Facultad de Ciencias Sociales y Educación en el año 2000 fueron posibles y que al cierre del año 2012, la calidad en la Universidad de Cartagena también está representada por el sello editorial de la Revista Palobra, palabra que obra.

\section{FUENTES}

Acevedo Puello, Rafael. “Apuntes acerca de la ciudadanía política en el Caribe Colombiano: El pensamiento educativo de principios del siglo XX", Revista Palobra, palabra que obra. No. 9, (2008): 81-101.

Agar Corbinos, Lorenzo. “Migraciones posmodernas en Chile: Reflexiones sobre cohesión social y pluralidad cultural, Revista Palobra, palabra que obra No. 12 (2011): 108-129.

Alayon, Norberto y María Lorena Molina. “La desigualdad social: Desarrollos y Desafíos del Trabajo Social desde la re conceptualización en América Latina", Revista Palobra, palabra que obra No. 9 (2008): 35-50.

Amezquita López, Julio y Erik Baldovino Villacop. "Prospectiva industrial de las mini cadenas productivas hortofrutícolas de Bolívar", Revista Palobra, palabra que obra. No.7(2007): 107-118.

Bolaño, Carmen y Hernández, Javier."Algunos elementos de la construcción de 'lo universitario' por los estudiantes de la Universidad de Cartagena", Revista Palobra, palobra que obra. No 1 (2000): 87-97.

Bonilla Vélez, Gloria. “Bellas, Casadas, madres y solteras, imágenes femeninas en el Caribe colombiano a comienzos del siglo XX", Revista Palobra, palabra que obra, palabra que obra.No. 1 (2000): 66-78.

Bonilla, Gloria. “La lucha de las mujeres en América latina: Ciudadanía y Derechos”, Revista Palobra, palabra que obra. No. 9, (2008): 45-59.

Bonilla Vélez, Gloria, Pilar Morad de Martínez y Anatali Oquendo Lorduy. "Feminidades y maternidades en Cartagena de indias: Entre las innovaciones y los conflictos", Revista Palobra, palabra que obra. No. 10, (2009): 57-72.

Cabrales, Carmen. Editorial. Revista Palobra, palabra que obra. No.1 (2000): 5-7.

Cogollo, Kenia, Lewis Leon y Berena Vergara Serpa. “Cartagena de Indias y la asociatividad como estrategia competitiva de sobrevivencia en el desarrollo local", Revista Palobra, palabra que obra. No. 6 (2005): 28-41.

De León Herrera, Rina. "Los estudios de pobreza urbana”, Revista Palobra, palabra que obra. No. 9 (2008): 78-98; Camilo Rey Sabogal, “Aproximaciones teóricas y empíricas a la relación de causalidad entre desigualdad y crecimiento: Un análisis para Colombia. 19852006". 164-184. 
Durango Rueda, Orlando. “Estableciendo rastros para la construcción de una persona activa de Derechos. El cuerpo de la violencia en la historia de los Montes de María", Revista Palobra, palabra que obra. No. 9(2008): 121-136.

Gutiérrez, Edgar. "Elementos para una filosofía del universo simbólico y ciudadanía en el contexto de Colombia yLatinoamérica", Revista Palobra, palabra que obra. No 2 (2001): $12-27$.

Hernández García, Javier. "Una teoría retórica de la Sociedad", Revista Palobra, palabra que obra. No. 6 (2005): 104-122.

Hernández, Javier. "Formas y modos del trabajo en la hacienda tradicional Monte mariana. (1930-1960)", Revista Palobra, palabra que obra. No. 9, (2008): 102-125.

Jiménez Torrado, Carmenza y Leydi Perneth Pareja “Exigencias metodológicas del abordaje de la realidad social desde una perspectiva de género." Una experiencia en construcción, Revista Palobra, palabra que obra. No 9 (2008): 99-120.

Masullo Jiménez, Juan. "La Genética del discurso del desarrollo", Revista Palobra, palabra que obra. No. 9 (2008): 84-103.

Mejía,Marco Raúl. "Hacia una pedagogía del conflicto", Revista Palobra, palabra que obra. No 2 (2001): 60-82.

Morad de Martínez, María del Pilar.“La construcción sociocultural de la maternidad y la paternidad en Cartagena de indias", Revista Palobra, palobra que obra. No 1 (2000): 16-24.

Morad de Martínez, María del Pilar. “Familias: espacios con posibilidades de construir y deconstruir ideas de equidad entre los géneros", Revista Palobra, palabra que obra. No. 3 (2002): 123-131.

Mosquera Rosero-Labbé, Claudia.“Sufrir el desplazamiento forzado para conocer los Derechos: impactos del desplazamiento forzado en mujeres afrocolombianas residentes en Bogotá", Revista Palobra, palabra que obra. No. 6 (2005): 42-53.

OnetoPiazze, Leonardo. "De las representaciones discursivas al orden del discurso: Una perspectiva discursiva de la intervención del Trabajo Social", Revista Palobra, palabra que obra. No. 9 (2008): 27-41.

Ospina Bozzi, Carlos. "Festival ver ciencia Caribe: Un modelo de apropiación social del conocimiento científico regional", Revista Palobra, palabra que obra. No. 7 (2006): 38-50.

Pabon Patiño, Morelia. "Instauración de la Formación ambiental en la Universidad Colombiana", Revista Palobra, palabra que obra. No. 7 (2006): 51-75.

Pérez Mendoza, Liliana. "Las Políticas e intervenciones sociales, algunas consideraciones ética", Revista Palobra, palabra que obra. No. 11 (2010): 24-38.

Pérez Montfort, Ricardo y Gabriela Pulido Llano. “Cultura Cubana y Medios de Comunicación en México, 1920-1950", Revista Palobra, palabra que obra. No. 12 (2011): 16-35.

Pimienta Betancourt, Alejandro. "La configuración de la identidad local en la diversidad cultural: El Caso de Caucasia", Revista Palobra, palabra que obra. No. 9 (2008): 60-77.

Piñeres de la Ossa, Dora. “El ingreso de la mujer a la universidad, entre discursos y debates: el caso de Paulina Beregoff. La primera universitaria en Colombia", Revista Palobra, palabra que obra No. 3 (2002): 19-32.

Piñeres de la Ossa, Dora. “El movimiento renovador estudiantil y las reformas universitarias en Colombia, 1920-1930", Revista Palobra, palabra que obra. No. 1 (2000): 78-87. 
Piñeres de la Ossa, Dora. "La pedagogía activa en los claustros de la Universidad de Cartagena, la presencia del pedagogo alemán Carl Glockner", Revista Palobra, palobra que obra. No. 5(2004): 19-27.

Puyana, Yolanda. "Cómo se convierten en mujeres las niñas del norte de Bolívar", Revista Palobra, palabra que obra. No. 1 (2000):25-39.

Rey Sinning, Edgar. "Las nuevas liturgias patrióticas: Celebraciones en Santa marta, con ocasión de la elección y posesión de tres presidentes neogranadinos", Revista Palobra, palabra que obra. No. 7 (2006): 76-91.

Rodríguez González, Hortensia Naizara. “La violencia intrafamiliar en Cartagena: un asunto de mentalidades", Revista Palobra, palabra que obra. No. 6 (2005): 71-84.

Solano de las Aguas, Sergio Paolo. "Imprentas, Tipógrafos y estilos de vida en el Caribe Colombiano. 1850-1930", Revista Palobra, palabra que obra. No.9 (2008): 126-145.

Valencia,Mabel, y Carlos Ospina. “El abordaje de la problemática de la explotación sexual infantil en Cartagena", Revista Palobra, palobra que obra., No. 6 (2005): 137-153.

De la Ossa, Dora Piñeres; Hernández García, Javier; Simancas Mendoza, Estella."PALOBRA, palabra que obra". Revista Historia de la Educación Latinoamericana. Vol. 15 No, 20, (2013): 135 - 150. 